\title{
VISTA: A Promising Target for Cancer Immunotherapy?
}

\author{
Marco Tagliamento (iD) 1,2 \\ Elisa Agostinetto 3,4 \\ Roberto Borea (iD ${ }^{2,5}$ \\ Mariana Brandão 3 \\ Francesca Poggio 6 \\ Alfredo Addeo (iD ${ }^{7}$ \\ Matteo Lambertini (iD ${ }^{2,8}$ \\ 'Department of Medical Oncology, \\ Medical Oncology 2, IRCCS Ospedale \\ Policlinico San Martino, Genova, Italy; \\ ${ }^{2}$ Department of Internal Medicine and \\ Medical Specialties (Di.M.I.), University of \\ Genova, Genova, Italy; ${ }^{3}$ Institut Jules \\ Bordet and Université Libre de Bruxelles \\ (U.L.B), Brussels, Belgium; ${ }^{4}$ Medical \\ Oncology and Hematology Unit, IRCCS \\ Humanitas Clinical and Research Center \\ and Humanitas University, Milan, Italy; \\ ${ }^{5}$ Department of Medical Oncology, \\ Medical Oncology I, IRCCS Ospedale \\ Policlinico San Martino, Genova, Italy; \\ ${ }^{6}$ Breast Unit, IRCCS Ospedale Policlinico \\ San Martino, Genova, Italy; ${ }^{7}$ Oncology \\ Department, University Hospital of \\ Geneva, Geneva, Switzerland; \\ ${ }^{8}$ Department of Medical Oncology, UOC \\ Clinica di Oncologia Medica, IRCCS \\ Ospedale Policlinico San Martino, \\ Genova, Italy
}

Correspondence: Marco Tagliamento Department of Internal Medicine and Medical Specialties (Di.M.I.), University of

Genova, Genova, Italy

Tel +39010 5558902

Email marco.tagliamento@edu.unige.it

\begin{abstract}
Agents targeting the B7 family co-inhibitory receptors cytotoxic T-lymphocyte antigen-4 (CTLA-4) and programmed cell death protein-1 (PD-1), or its ligand (PD-L1), have a pivotal role in clinical practice. V-domain Ig suppressor of T-cell activation (VISTA) is a protein highly conserved between species, with a similar amino acid sequence to the B7 family members, characterized by a particularly structural homology to PD-1. It has been counted as an emerging target within the list of novel targetable immune checkpoints in oncology. Physiologically, VISTA exerts a regulatory function on the immune system at several levels, particularly by modulating T cells activation. Its altered activity plays a role in many autoimmune diseases, and its expression has been found to be prognostically implicated in different cancer types in preclinical models. We hereby present the main evidence on the value of VISTA as an immune checkpoint in solid and hematological malignancies. We also review its value as a potential target for cancer immunotherapy, by reporting the results of Phase I and II clinical trials assessing the use of drugs targeting VISTA. The complexity of its pathway, along with some unclear biological aspects concerning its molecular interactions, currently represent a limit to the applicability of VISTA as an effective biomarker for immunotherapy in oncology. A deeper characterization of this immune checkpoint may help defining its value within immune signatures of solid and hematological malignancies, and to design future therapeutic strategies.
\end{abstract}

Keywords: VISTA, immune checkpoint, immunotherapy, cancer, biomarker

\section{Introduction}

\section{Immune Checkpoint Signaling and Cancer}

Immune suppression is one of the escape mechanisms adopted by cancer to overcome immune surveillance. Multiple mechanisms contribute to suppress immune cells that populate the tumor's microenvironment, both through signaling and metabolic processes. ${ }^{1}$ The discovery of immunoreceptors and the definition of their role in modulating the immunosurveillance have become a crucial hallmark in the treatment of cancer. Tumor cells work by upregulating the role of inhibitory immunoreceptors and by downregulating that of stimulatory immunoreceptors. ${ }^{1}$ The development of immune checkpoint inhibitors (ICIs) has represented a cornerstone in oncology. The rationale of their activity is the restoration of the immune competence by removing the breaks to antitumor immune responses, particularly by blocking inhibitory signaling pathways. ${ }^{2}$

To date, agents targeting the B7 family co-inhibitory receptors cytotoxic T-lymphocyte antigen-4 (CTLA-4) and programmed cell death protein-1 (PD-1) have hired a pivotal role in clinical practice. $^{2}$ 
Novel pathways involving other inhibitory immunoreceptors, particularly those eliciting $\mathrm{T}$ cell suppression, have been discovered and studied to serve as predictive biomarkers in preclinical and clinical models. ${ }^{1,3-8}$ Among them, lymphocyte-activation gene 3 (LAG3), ${ }^{9}$ programmed cell death protein-ligand 2 (PD-L2), ${ }^{10} \mathrm{~B}$ and T lymphocyte attenuator (BTLA), ${ }^{11}$ T-cell immunoglobulin and mucin domain 3 (TIM3), ${ }^{12,13} \mathrm{~T}$ cell immunoglobulin and ITIM domain (TIGIT), ${ }^{14} \mathrm{~B} 7-\mathrm{H} 3,{ }^{15} \mathrm{~B} 7-\mathrm{H} 4,{ }^{16}$ and V-domain Ig suppressor of T-cell activation (VISTA) ${ }^{17}$ have been described as emerging targets in the list of immune checkpoints. Moreover, neoantigens represent a promising tool for immunotherapy in oncology. They are new peptide sequences (neoepitopes) generated by non-synonymous somatic mutations, strictly tumour specific thus identified by T-cells as non-self-proteins, and detectable by genomic-based computational tools. Actually, personalized vaccines and tumour infiltrating lymphocytes (TILs)-based adoptive T-cell therapy have been investigated as targeting strategies. ${ }^{18}$

In this review, we explore the role of VISTA in the landscape of cancer immunotherapy.

\section{VISTA: Genomic and Molecular Structure}

Human VISTA is a 279-amino acid protein with similar sequence to the B7 family of ligands and receptors. It is structured with an extracellular domain (162-amino acid), a transmembrane domain (21-amino acid), and a cytoplasmic domain (96-amino acid). ${ }^{19}$ It is also known as differentiation of embryonic stem cells 1 (Dies1), c10orf54, VSIR, Gi24, B7-H5, SISP1, DD1 $\alpha$ and PD-1 homolog (PD-1H) ${ }^{20}$ Its transcript is coded by the gene Vsir, located into an intron of the $\mathrm{CDH} 23$ gene on chromosome 10 (10q22.1). ${ }^{19}$

VISTA is highly conserved between species showing $76 \%$ identity between mouse and human (its cytoplasmic tail shares up to $91 \%$ identity), and the highest sequence identity between mouse and zebrafish genomes. The closest homolog to VISTA within the B7 family is PD-L1, which shares $22 \%$ sequence identity. VISTA also resembles to CD28 and CTLA-4 but does not have a classic ITIM/ITAM motif as compared to other B7 co-receptors. Moreover, it does not include a $\operatorname{IgC}$ domain but only a single $\operatorname{IgV}$ domain, and it includes a protein kinase $\mathrm{C}$ binding site and a proline-rich motif that can serve for the interaction with other molecules. ${ }^{20}$ Thus, the role of VISTA as either a ligand, a receptor, or both, has been assumed. ${ }^{19}$ Both mechanisms could contribute to the overall role of VISTA. ${ }^{3}$ Figure 1 depicts the molecular structure of VISTA protein and its function at a cellular level.

\section{Physio(Patho)Logical Function of Vista: in vitro and in vivo Models}

In mice, VISTA is expressed predominantly in hematopoietic tissues, such as the spleen, thymus, lymph node, and bone marrow (BM) ${ }^{21}$ The lung and the small intestine also show high levels of expression, presumably due to high leukocytes infiltration. In non-hematopoietic tissues, including brain, heart, muscle, testis, and placenta, lower levels of VISTA messenger RNA (mRNA) have been reported. $^{21}$ In mice, VISTA mRNA is expressed during the embryonic stem cell phase. Different studies suggest it plays a role in regulating the signaling of bone morphogenic protein 4 (BMP4), causing stem cell differentiation. ${ }^{22,23}$ Among the hematopoietic compartment, myeloid cells express the highest level of VISTA mRNA and of the corresponding protein. VISTA protein is also expressed on naïve CD4+ and CD8+ TCR $\alpha \beta$ T cells, NK cells, regulatory T cells (Treg), and TCR $\gamma \delta$. On the contrary, VISTA protein is not expressed on B cells. ${ }^{24}$ Similarly to mice protein, human VISTA protein is mainly expressed on hematopoietic tissues, especially in those with high infiltration of leukocytes. ${ }^{25}$ Bharaj et al characterized the expression of VISTA protein on healthy humans, finding high levels on $\mathrm{CD} 14+$ monocytes, low levels on CD3 + T lymphocytes and CD19+ B cells. ${ }^{26}$ In order to understand the mechanism of VISTA regulation, monocytes were cultured with different cytokines, and only IL-10 and IFN- $\gamma$ led to an upregulation of VISTA levels. $^{26}$

Currently, little is known about the function of VISTA within the immune system. VISTA regulates T cells function on hematopoietic cells and leukocytes, reducing their activity. The first evidence about VISTA role on $\mathrm{T}$ cell regulation was derived from autoimmune encephalomyelitis murine models. ${ }^{21}$ VISTA blockade increased $\mathrm{T}$ cellmediated immunity, suggesting the role of VISTA in controlling autoimmunity similarly to PD-L1 and PD-L2. ${ }^{21}$ In VISTA knockout (KO) mouse model, the deactivation of VISTA pathway led to spontaneous activation of CD4+ and $\mathrm{CD} 8+\mathrm{T}$ lymphocytes and chronic inflammation among multiple tissues. ${ }^{27}$ The inflammation is supported by the production of different cytokines, such as IFN- $\gamma$, TNF- $\alpha$, and IL-17A, that induce a Th1-polarizing 


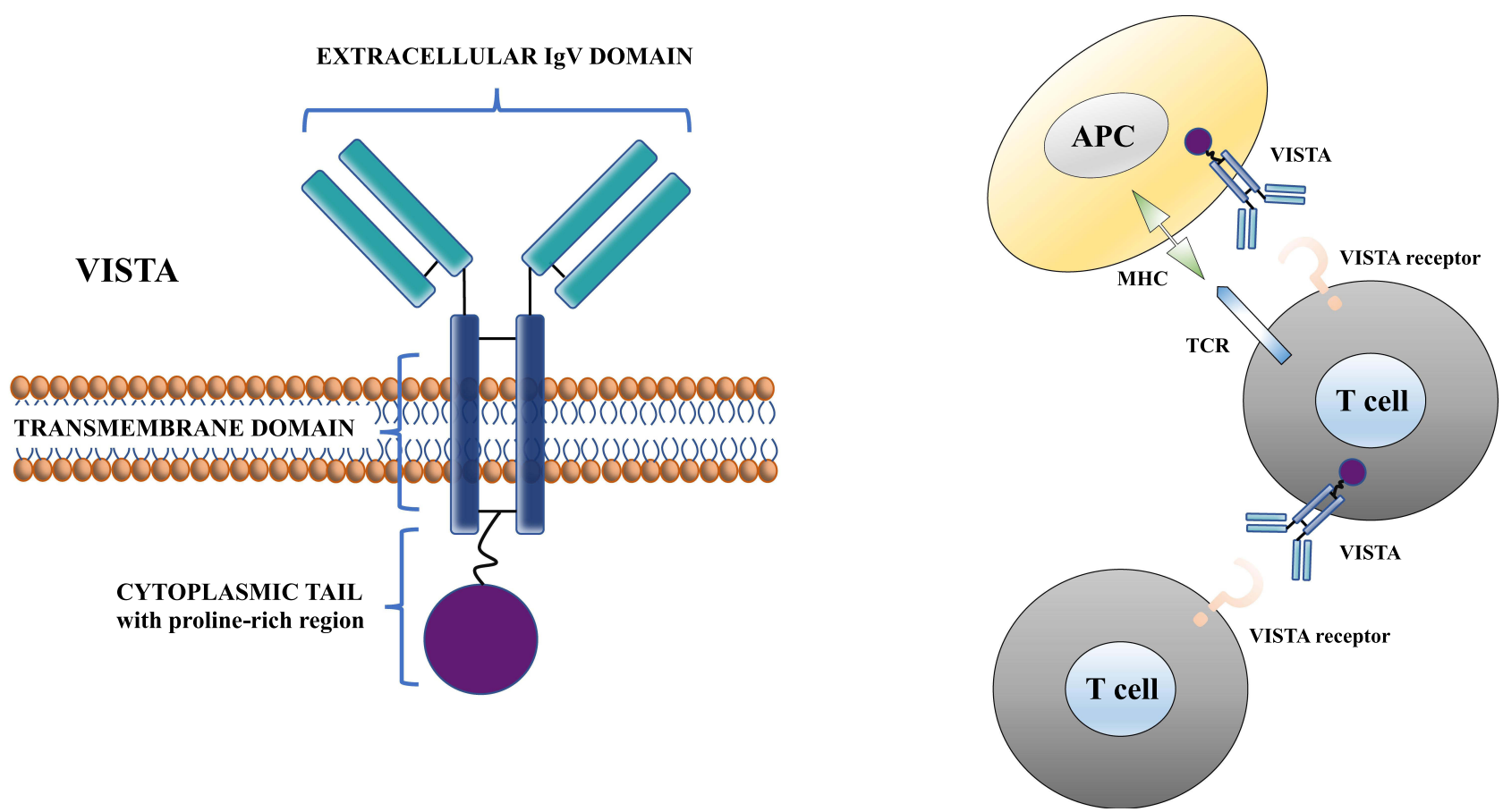

Figure I Molecular structure of VISTA protein and its function at a cellular level.

Notes: Vista plays a role as ligand on APCs and as receptor on T cells. Modified from Tagliamento M, Bironzo P, Novello S. New emerging targets in cancer immunotherapy: the role of VISTA. ESMO Open. 2019;4:e000683. ${ }^{17}$ @) 2020 The Authors. Creative Commons CC-BY-NC

Abbreviations: APC, antigen-presenting cell; TCR, T-cell receptor; MHC, Major Histocompatibility Complex.

phenotype. An interesting hypothesis is that VISTA KO may reduce the threshold of T-cell receptor (TCR) activation towards self-antigens. ${ }^{27}$

A Concanavalin A-induced acute hepatitis VISTA KO mouse model developed hepatitis of higher grade than wild-type mice control. Interestingly, approximately the same number of CD4+ and NKT cells were found in the liver. These findings support the role of VISTA in regulating $\mathrm{T}$ cell immune responses. ${ }^{28}$

In mouse models of graft-versus-host disease (GVHD), the use of the anti-PD-1H monoclonal antibody (mAb) MH5A prevented almost all mice from death. ${ }^{24}$ The same authors demonstrated that in GVHD mouse model treated with MH5A, the expansion of donor CD4+ and CD8+ T cells was significantly reduced, while any loss of CD19+ B cells was observed. ${ }^{29}$ These observations demonstrate that MH5A directly inhibits allo-reactive $\mathrm{T}$ cell expansion and its functionality.

To better understand the possible synergism between VISTA and PD-1, Liu et al investigated chronic inflammation and $\mathrm{T}$ cell activation in a single $\mathrm{KO}$ mouse model (VISTA and PD-1) and a in double VISTA/PD-1 KO mouse model. They demonstrated that VISTA pathway is not redundant with PD-1 pathway in controlling T-cell activation. $^{30}$

An in vivo experiment on VISTA deficient murine models of acute and chronic immune-complex mediated glomerulonephritis proved a reduction of neutrophil degranulation and, in vitro, VISTA-deficient neutrophils had low activity in response to immune complexes. This suggests that VISTA may be a potential target in immunecomplex mediated glomerulonephritis. ${ }^{31}$

Giant cell arteritis is an autoimmune disease induced by the failure of immune checkpoint regulation. Hid Cadena et al studied the role of VISTA in activating $\mathrm{T}$ cell in giant cell arteritis, reporting that VISTAdirected Ig failed to suppress Th1, and Th17, probably due to the reduced expression of VISTA on these cells. ${ }^{32}$

Finally, checkpoint regulators, such as PD-1/PD-L1 and CTLA-4, are important factors in inflammation of the central nervous system. In mouse models and in humans, VISTA is extensively expressed by microglia and less by endothelial cells. The in vivo expression of VISTA depends on the microglia activation; in multiple sclerosis lesion tissue his expression was low, whereas it was high in patients with Alzheimer's disease. ${ }^{33}$ 
The role of VISTA in regulating inflammation is not only based on $\mathrm{T}$ cell regulation, since VISTA can instead re-program macrophage biology. In vitro experiments demonstrated that VISTA agonists mAb were responsible for a reduction of lipopolysaccharide-induced IL-12p40, IL-6, CXCL2, and TNF, key pro-inflammatory mediators of endotoxin shock, supporting the hypothesis that VISTA is a negative checkpoint regulator which induces both tolerance and anti-inflammatory programs in macrophages. ${ }^{34}$

Little is known about the cognate receptor for VISTA and the mechanism of how VISTA regulates T cell activation. Nevertheless, it is known that VISTA plays an intrinsic and extrinsic role in regulating $\mathrm{T}$ cell activity. The intrinsic system implies an inhibition of $\mathrm{T}$ cell activation induced by phosphorylation of TCR. This is supported by in vitro evidences, where immobilized VISTA-ectodomain suppressed $\mathrm{T}$ cell activation. ${ }^{21,30}$ The extrinsic one is provided by antigen-presenting cells (APCs). On APCs, VISTA binds a coinhibitory receptor on $\mathrm{T}$ cells leading to a suppressive stimulus. In vitro data showed that VISTA deletion on both $\mathrm{T}$ cells and APCs, when cocultured, considerably increased the proliferation of $\mathrm{T}$ cells if compared with a single deficient VISTA cell, either T cells or APCs. ${ }^{28}$ Despite the demonstrations on the role of VISTA on $\mathrm{T}$ cells activation, VISTA ligand has yet to be clearly identified. Nevertheless, V-Set and Immunoglobulin domain containing 3 (VSIG-3), also known as Immunoglobulin Superfamily member 11 (IGSF11) and Brain-specific and Testis-specific Immunoglobulin Superfamily (BT-IgSF), has been reported to act as a coinhibitory stimulus to activated T-cell functions by bonding VISTA. VSIG-3 is highly expressed in colorectal cancers, hepatocellular carcinomas and intestinal-type gastric cancers. Further studies on VSIG-3/VISTA interaction may better elucidate this pathway. ${ }^{35}$

\section{Vista Expression in Cancer and Its Value as an Immune Checkpoint: Preclinical Data}

The role of VISTA in cancer immunity is complex, with most preclinical data suggesting its immunosuppressive function, thus supporting anti-VISTA treatments as a way to up-regulate immune response against cancer cells. ${ }^{20} \mathrm{We}$ hereby present the main preclinical findings on the value of VISTA as an immune checkpoint in solid and hematological malignancies. A detailed summary is reported in Table 1.

\section{Melanoma}

Melanoma is one of the most immunogenic tumor type, and various immunotherapeutic approaches have proven to be very effective in treating it. ${ }^{36}$ There are several reports evaluating VISTA expression and its role as immune checkpoint in melanoma.

Rosenbaum et al observed that VISTA promotes tumor onset in immunocompetent mouse melanoma models and that the mutant BRAF-regulated transcription factor (FOXD3) suppresses VISTA expression at the transcript level. ${ }^{37}$ Consistently, $\mathrm{Xu}$ et al, using B16-BL6 murine melanoma cells, showed that the inhibition of VISTA signaling induced an increased production of proinflammatory mediators and diminished the $\mathrm{T}$ cell-suppressive functions of myeloid-derived suppressor cells, thus confirming the immunosuppressive role of VISTA signaling in melanoma and supporting the rational to investigate VISTA as a potential therapeutic target. ${ }^{38}$

Further evidence on the role of VISTA in melanoma comes from a study conducted on mouse models inoculated with B16BL6 melanoma cells, conditioned with lowdose irradiation and treated with four doses of GVAX (a lethally irradiated granulocyte-macrophage colony stimulating factor-secreting allogeneic whole-cell melanoma vaccine) before the treatment with either VISTA or PDL1 inhibitors, or both. ${ }^{30}$ In this study, treatment with both VISTA and PD-L1 mAb significantly suppressed tumor growth and conferred survival advantage. ${ }^{30}$ This finding could indicate that VISTA and PD-L1/PD-1 are independent pathways in controlling tumor-specific $\mathrm{T}$ cell responses, and a combined therapeutic blockade can synergistically enhance the antitumor immunity.

Studies conducted on human melanoma samples are also available. Kakavand et al compared VISTA expression on melanoma biopsies collected from 16 patients who initially responded to treatment with ICIs and then progressed. ${ }^{39}$ Interestingly, a significant increase in the density of VISTA positive lymphocytes was observed from pre-treatment biopsies to progression, as well as an increased expression of tumor PD-L1. ${ }^{39}$ This increase in VISTA expression is interesting and may represent a potential therapeutic target in metastatic melanoma patients, particularly in those progressing to anti-PD-1 therapy, and warrants further assessment in clinical trials. 


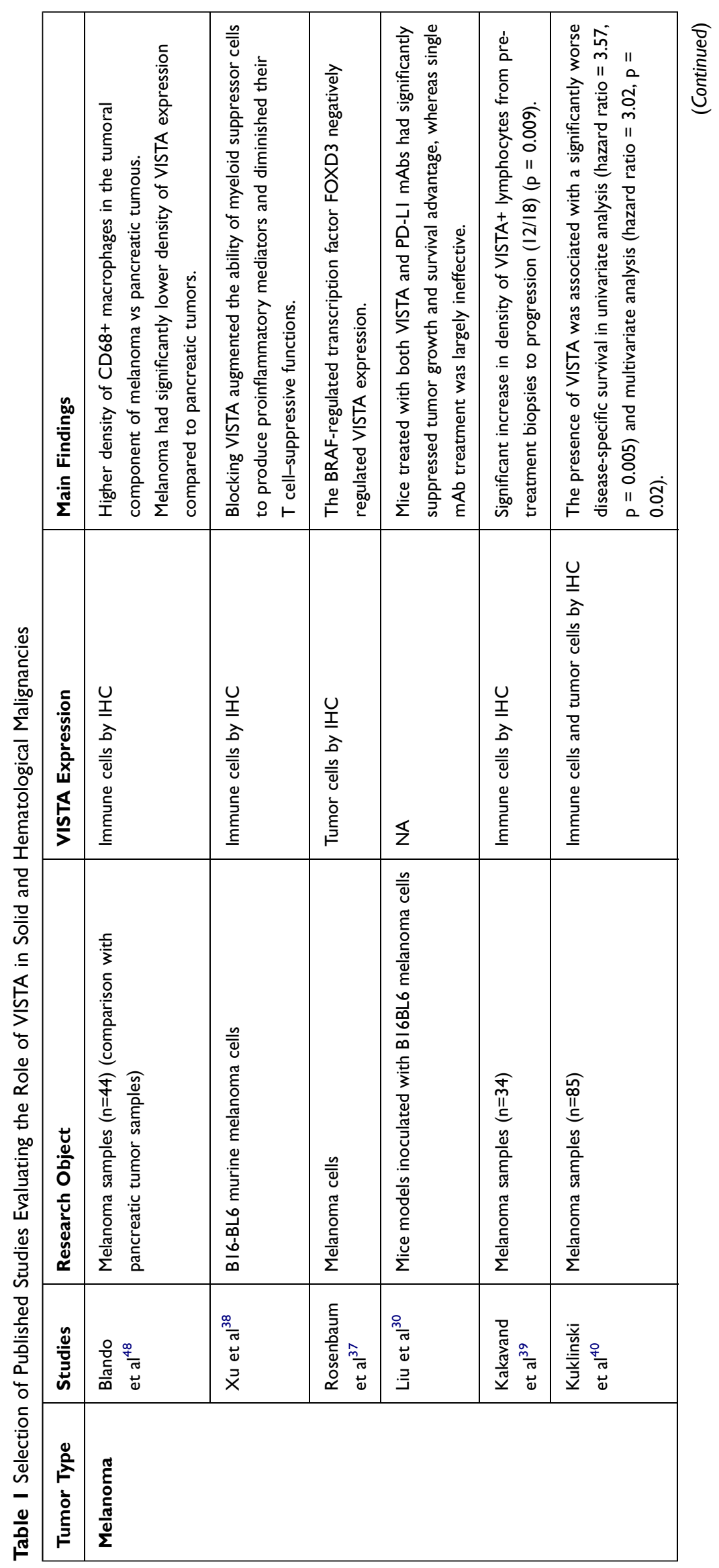




\begin{tabular}{|c|c|c|c|c|c|}
\hline 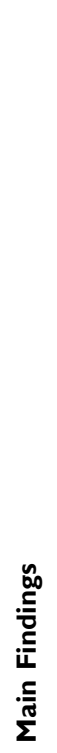 & 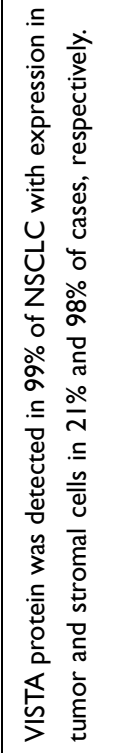 & 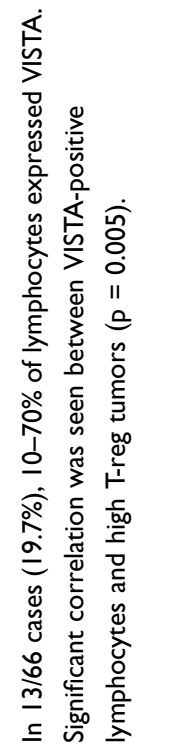 & 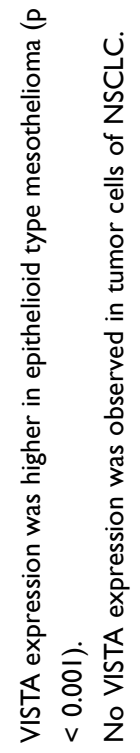 & 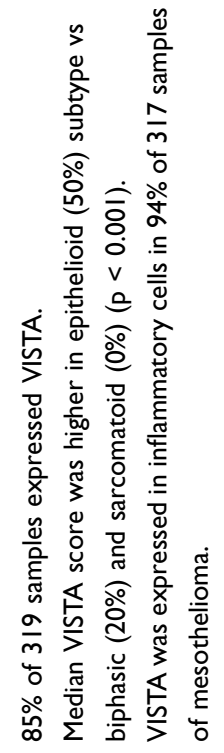 & 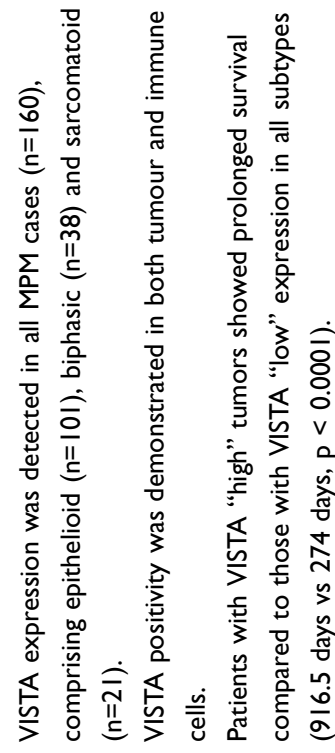 \\
\hline 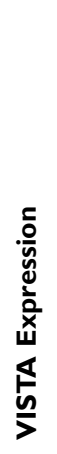 & 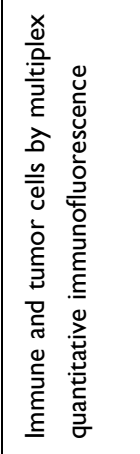 & 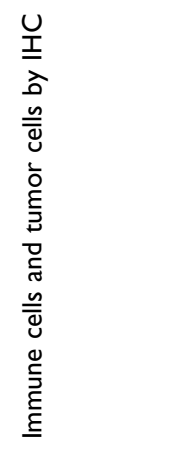 & 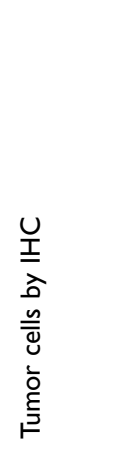 & 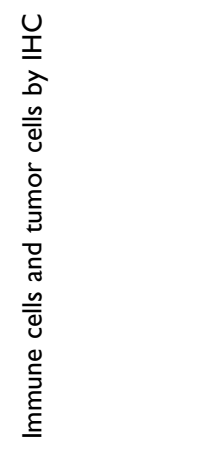 & 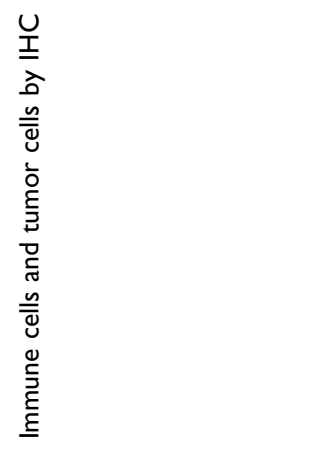 \\
\hline 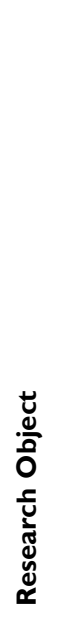 & 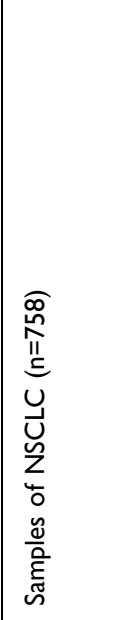 & 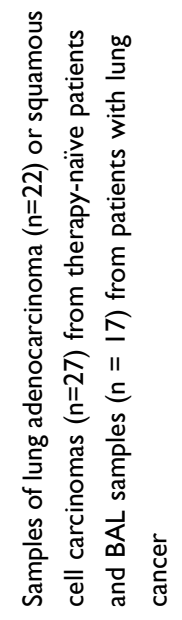 & 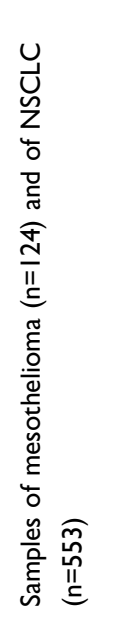 & 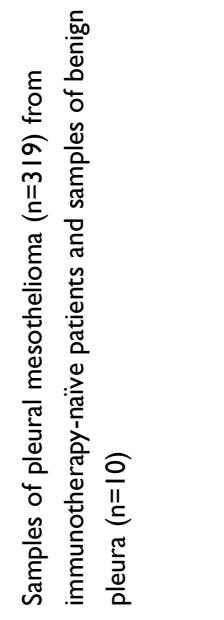 & 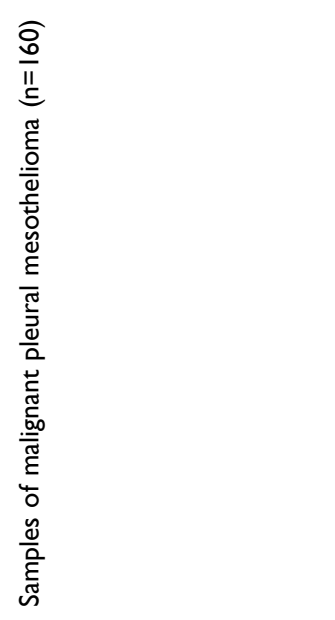 \\
\hline 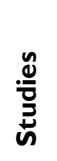 & 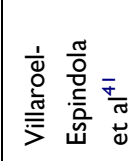 & 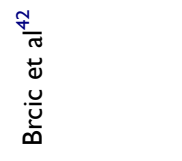 & 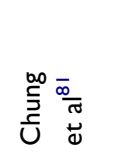 & 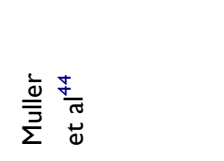 & 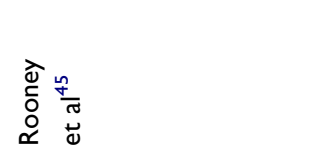 \\
\hline 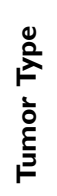 & 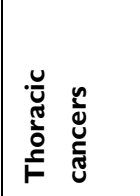 & & & & \\
\hline
\end{tabular}




\begin{tabular}{|c|c|c|c|c|}
\hline 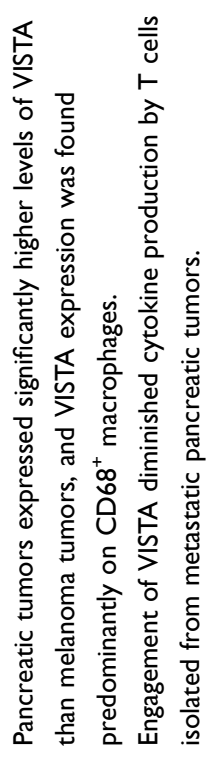 & 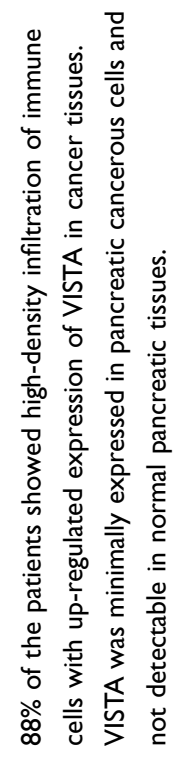 & 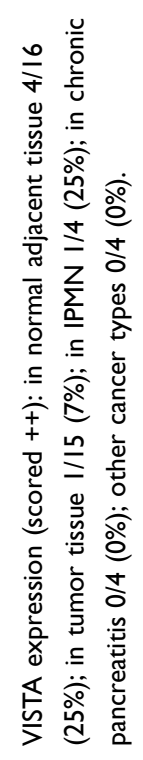 & 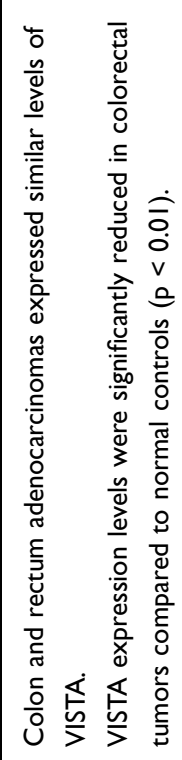 & 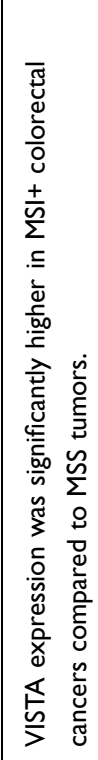 \\
\hline 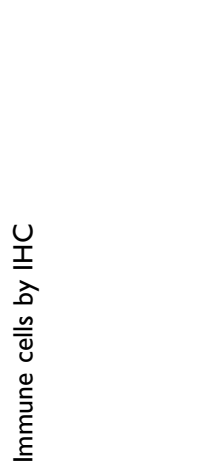 & 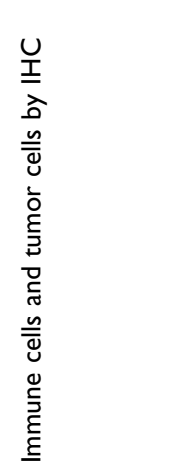 & 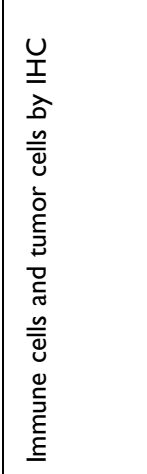 & 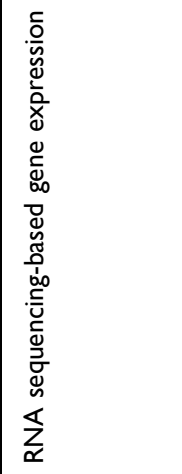 & 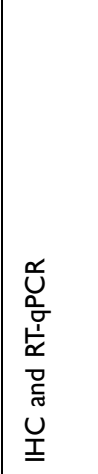 \\
\hline 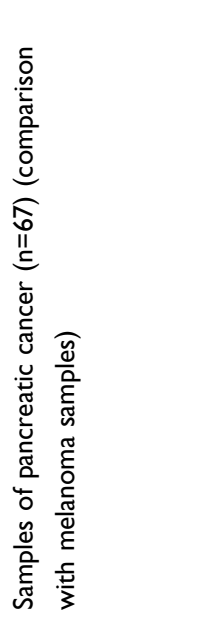 & 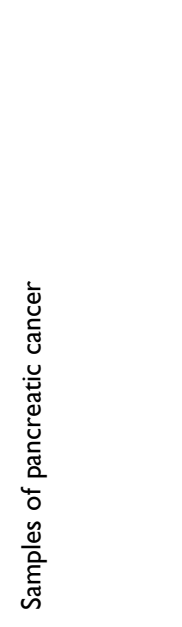 & 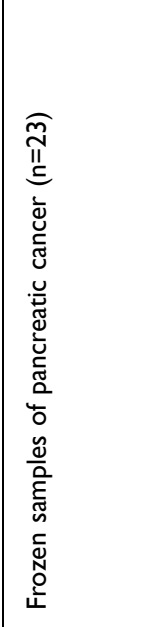 & 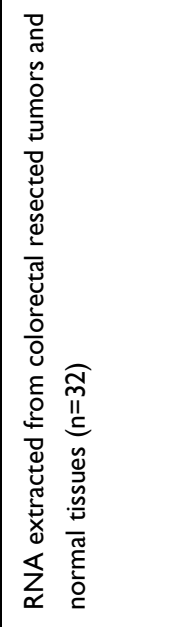 & 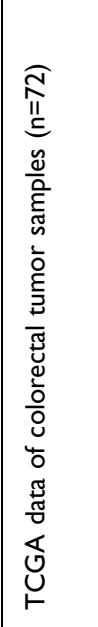 \\
\hline 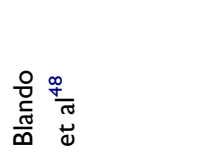 & 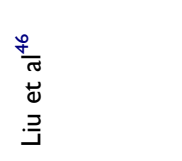 & 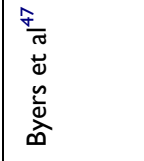 & 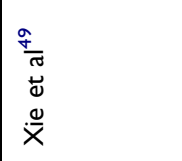 & 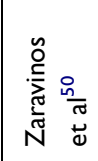 \\
\hline 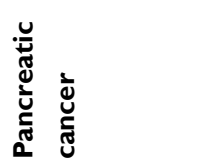 & & & 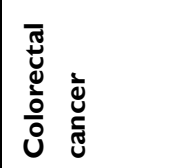 & \\
\hline
\end{tabular}




\begin{tabular}{|c|c|c|c|c|c|c|c|}
\hline 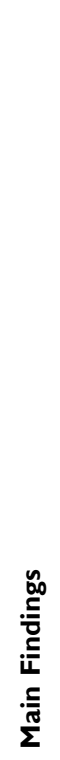 & 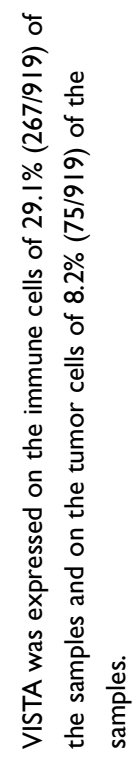 & 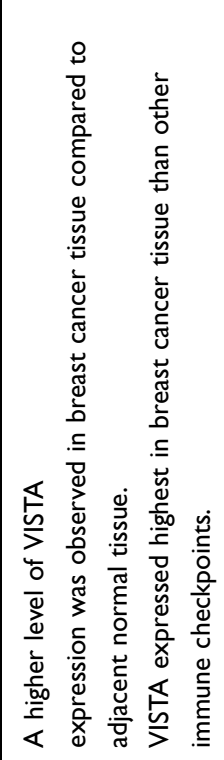 & 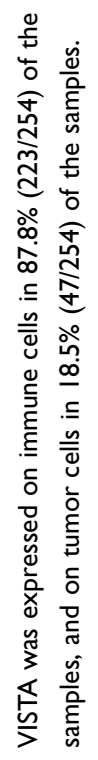 & 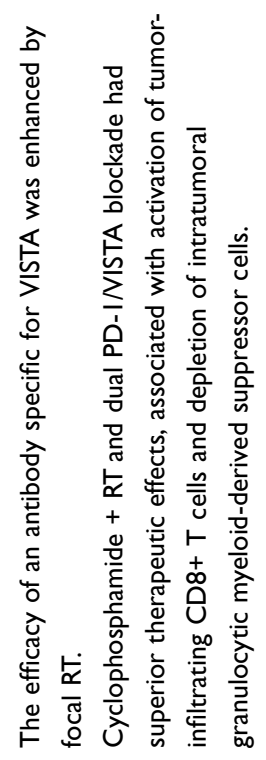 & 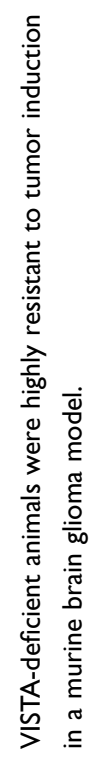 & 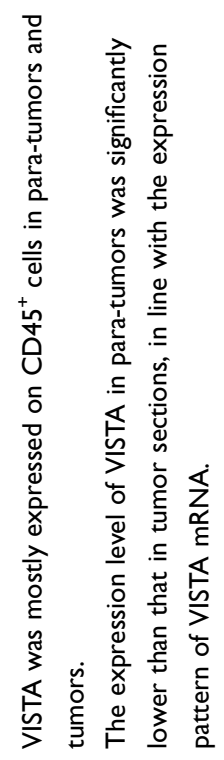 & 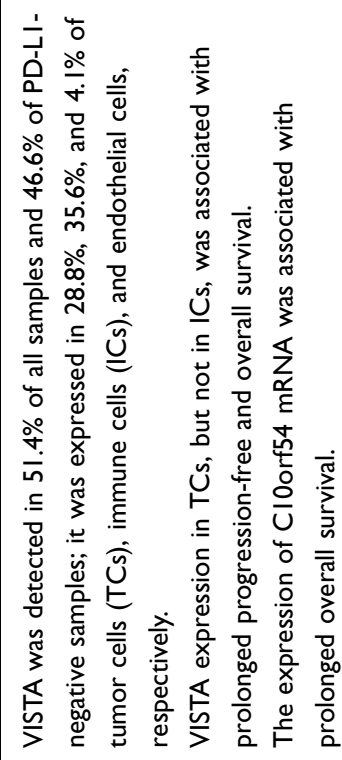 \\
\hline 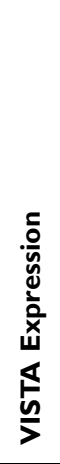 & 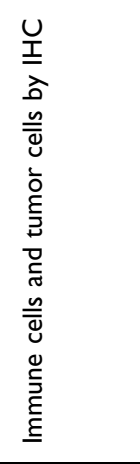 & 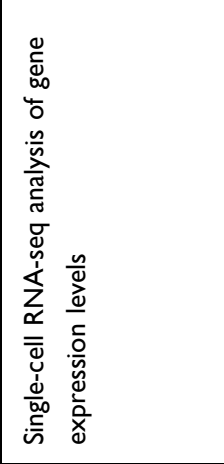 & 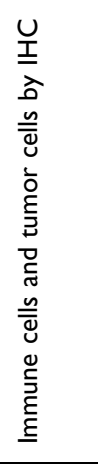 & $\S$ & $\S$ & 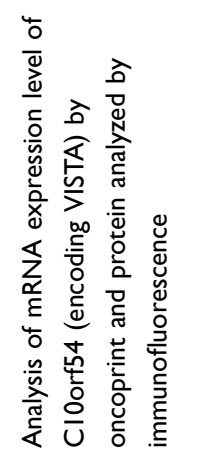 & 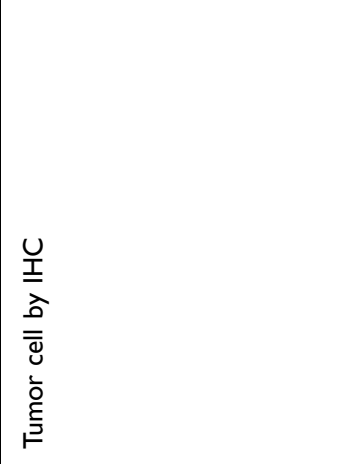 \\
\hline 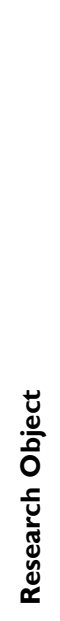 & 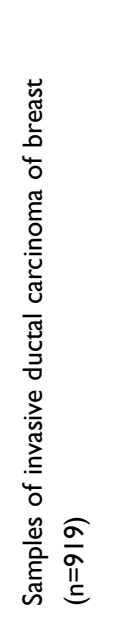 & 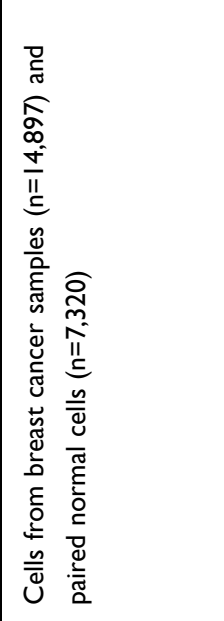 & 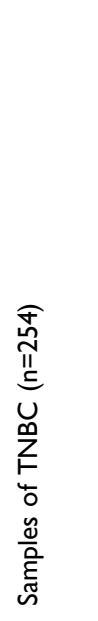 & 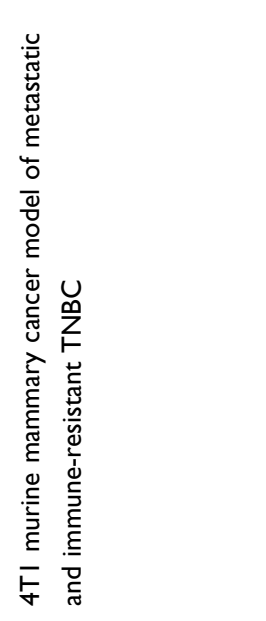 & 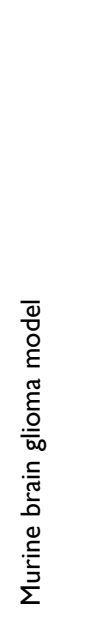 & 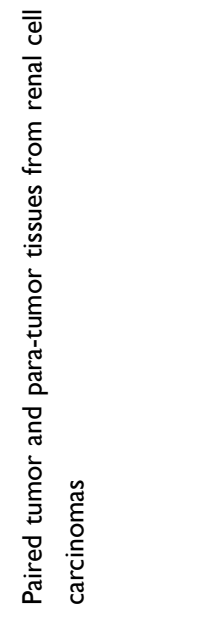 & 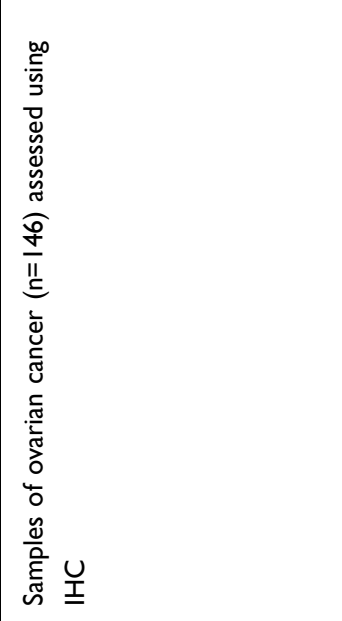 \\
\hline 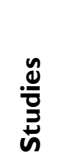 & 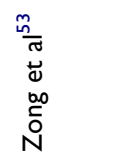 & 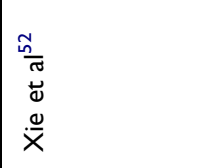 & 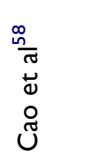 & 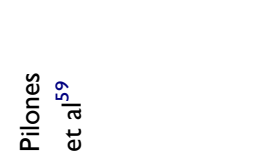 & 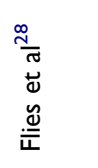 & $\begin{array}{l}\frac{0}{0} \\
\frac{0}{0} \\
0 \\
00 \\
\frac{0}{1} \\
\frac{1}{1}\end{array}$ & 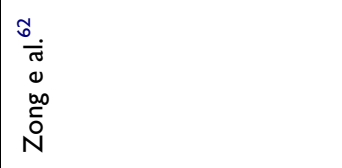 \\
\hline 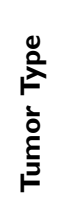 & 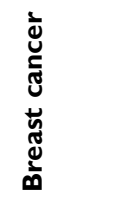 & & & & 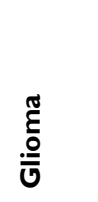 & 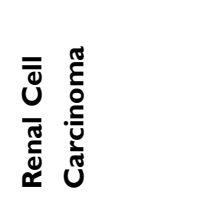 & 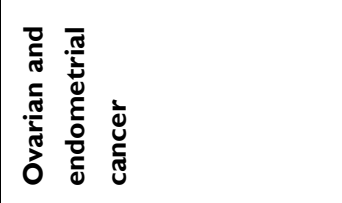 \\
\hline
\end{tabular}




\begin{tabular}{|c|c|}
\hline 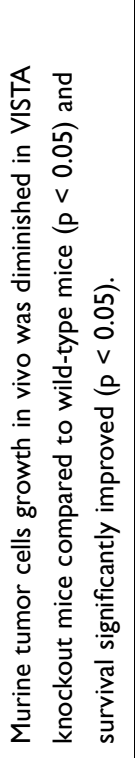 & 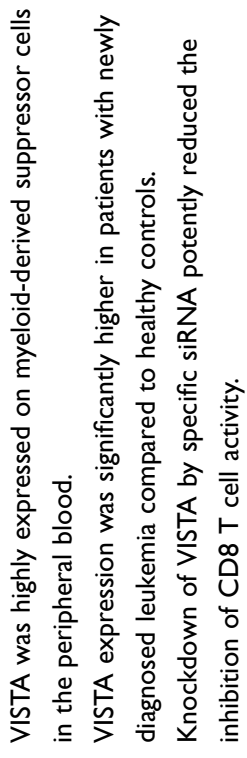 \\
\hline 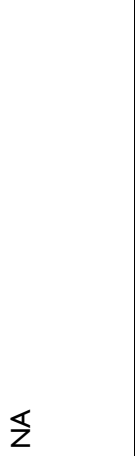 & 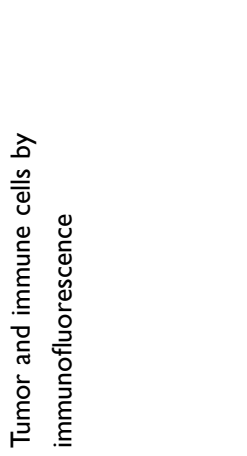 \\
\hline 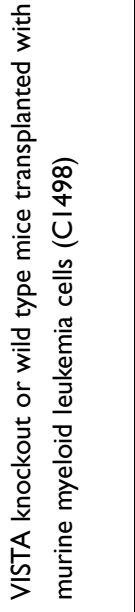 & 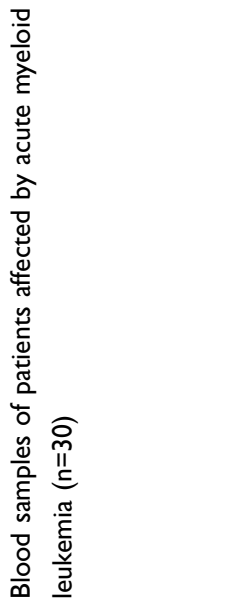 \\
\hline 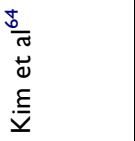 & 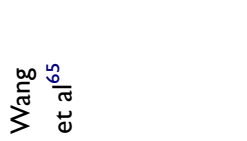 \\
\hline 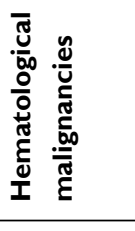 & \\
\hline
\end{tabular}

In addition, there is evidenceof VISTA as a negative prognostic factor in melanoma. Kuklinski et al, evaluating 85 specimens of primary melanoma at different pathologic stage, observed that VISTA expression on tumorinfiltrating inflammatory cells was associated with worse disease-free survival both at univariate (hazard ratio [HR] $=3.57, \mathrm{p}=0.005)$ and multivariate $(\mathrm{HR}=3.02, \mathrm{p}=0.02)$ analyses. ${ }^{40}$

\section{Lung Cancer}

Several studies support the role of VISTA as an inhibitory immune checkpoint in lung cancer. VillarroelEspindola et al performed localized measurements of VISTA protein on 758 samples of stage I to IV nonsmall-cell lung cancer (NSCLC), and detected VISTA protein expression in $99 \%$ of specimens with a predominant expression in stromal cells, rather than tumor cells ( $98 \%$ and $21 \%$ of cases, respectively). ${ }^{41}$ The level of VISTA expression was associated with PD$\mathrm{L} 1$ and PD-1, and to CD8+ $\mathrm{T}$ cells and CD68+ macrophages levels, with a higher expression in $\mathrm{T}$ lymphocytes and in cytotoxic $\mathrm{T}$ cells compared to macrophages and T-helper cells, respectively. ${ }^{41}$ In a study led by Brcic et al, in roughly $20 \%$ of 49 analyzed resected lung cancer tissues, $10 \%$ to $70 \%$ of lymphocytes expressed VISTA. No correlation was observed between PD-L1 tumor-cell expression and PD-1 or VISTA expression on lymphocytes. ${ }^{42}$ Interestingly, a significant correlation was seen between VISTA-positive lymphocytes and high T-regulatory cells in tumor samples, indicating a potential influence of VISTA on immunosuppressive cells. ${ }^{42}$

\section{Mesothelioma}

Data extracted from The Cancer Genome Atlas Study showed that pleural mesothelioma displays the highest expression of VISTA among all cancers. ${ }^{43}$

In a study by Muller et al, VISTA was analyzed by immunohistochemistry on tumor and inflammatory cells from 319 immunotherapy-naive pleural mesotheliomas (254 epithelioid, 24 biphasic and 41 sarcomatoid) and 10 specimens of benign pleura. All benign tissues expressed VISTA, while $85 \%$ of 319 tumoral samples expressed VISTA. Median VISTA score was significantly higher in epithelioid vs biphasic and sarcomatoid. On multivariate analysis, VISTA expression in mesothelioma was associated with better overall survival (OS), contrary to what was found on regard to PD-L1 expression. ${ }^{44}$ 
In a study by Rooney et al, VISTA expression on both tumor and immune cells was detected in all 160 malignant pleural mesothelioma samples evaluated, independently by histology, and its detection was correlated with a better OS. $^{45}$

\section{Pancreatic Cancer}

Pancreatic cancer is traditionally associated with a poor prognosis, and to date several therapeutic approaches including immunotherapy have failed to achieve any significant benefit in the last decades. Liu et al observed that VISTA was expressed in pancreatic cancer, predominantly in immune cells infiltrating the tumor tissue (as observed in $88 \%$ of 52 analyzed patients), while it was minimally expressed on cancer cells and not detectable in normal pancreatic tissues. ${ }^{46}$ On the contrary, Byers et al showed that, using frozen samples from resected pancreatic cancers, VISTA expression was restricted to normal pancreas tissue, while its expression was downregulated in tumor tissue, suggesting that loss of VISTA signal could prompt immune evasion of pancreatic cancer. ${ }^{47}$ These controversial findings definitely underline the need for further investigation in this setting. Of note, pancreatic cancer is a peculiar tumor type, with particular characteristics, starting from its tumor microenvironment, consisting in a thick structure composed of fibrous connective tissue populated with myeloid cells and some spared lymphoid components. Blando et al performed a comprehensive analysis of the immune tumor microenvironment of 67 samples of pancreatic cancer, and compared it to the immune microenvironment of 44 melanoma samples. ${ }^{48}$ As expected, pancreatic tumor had minimal to moderate infiltration of CD3, CD4, and CD8 T cells; total CD68+ macrophages did not significantly differ between melanoma and pancreatic cancer, but a higher density of CD68+ macrophages was found in the tumoral component of melanoma compared with pancreatic tumors. Interestingly, despite lower density of CD68+ macrophages, pancreatic tumors had significantly higher density of VISTA expression compared to melanoma tumors. ${ }^{48}$ Moreover, the engagement of the VISTA inhibitory pathway resulted in a greater decrease in CD8 $+\mathrm{T}$ cell responses compared to that achieved by the engagement of PD-L1 pathway, and in a diminished cytokine production by $\mathrm{T}$ cells isolated from metastatic pancreatic tumors. ${ }^{48}$ These data seem to support the role of VISTA as a crucial inhibitory checkpoint molecule and, consequently, as a potential immunotherapeutic target in pancreatic cancer.

\section{Colorectal Cancer}

Several studies have been published on VISTA expression in colorectal cancer. Xie et al analyzed, by RNAsequencing, VISTA gene expression of colorectal tumors and normal tissue resected from 32 patients, and observed that colon and rectum adenocarcinomas expressed similar levels of VISTA. The expression, however, was significantly reduced in colorectal tumors compared to normal controls. ${ }^{49}$ This finding might suggest an immunosuppressive role of VISTA in colorectal cancer. Interestingly, Zaravinos et al showed that VISTA had a significantly higher expression in microsatellite unstable (MSI) compared to microsatellite-stable (MSS) colorectal tumors. ${ }^{50}$ These results add further potential explanations among the reasons why MSI tumors tend to have better response to immunotherapy, compared to MSS ones, and support further investigation on VISTA as a possible therapeutic target in colorectal cancer.

\section{Breast Cancer}

There is a limited number of studies focusing on VISTA expression in breast cancer compared to other tumor types, mainly because breast cancer is traditionally considered a "cold" tumor from an immunological point of view. ${ }^{51}$ Nonetheless, a single-cell RNA-sequencing analysis of gene expression levels of immune checkpoint molecules (including VISTA) from almost 15,000 cells of a breast cancer sample and from roughly 7,000 paired normal cells, proved that breast cancer tissue had higher levels of VISTA expression compared to adjacent normal tissue. ${ }^{52}$ VISTA expression was correlated with pathological grade (I-II vs III; $\mathrm{p}=0.001)$, lymph node status $(\mathrm{p}=0.045)$, tumor subtype (luminal, HER $2+$ and basal-like; $\mathrm{p}<$ $0.001)$ and PD-1 ( $=0.038)$, while it was not correlated with the expression of other immune checkpoints (like PDL1, TIGIT, TIM3, LAG3) in breast cancer tissue. ${ }^{52}$

The first study assessing the expression of VISTA by immunohistochemistry on 919 samples of invasive ductal carcinoma of the breast showed that VISTA was expressed on immune cells and on tumor cells in $29 \%$ and $8 \%$ of samples, respectively. ${ }^{53}$ As expected, VISTA was more frequently observed in oestrogen receptor (ER) and progesterone receptor (PgR) negative, HER2-positive, poorly differentiated tumors, and was associated with PD-1, PDL1, stromal CD8, and TILs expression. This might be 
consistent with the fact that ER-negative breast tumors are known to be more immunogenic, with higher genomic instability compared to other subtypes, and with stromal TILs, having a strong prognostic and predictive value in this subtype. ${ }^{54-57}$ Subsequent studies investigating the role of VISTA in breast cancer focused mainly on triplenegative subtype (TNBC). Cao et al evaluated the expression of VISTA in a cohort of 254 patients with early stage untreated $\mathrm{TNBC}$, and observed that $88 \%$ and $18 \%$ of samples expressed VISTA in the immune cells and in tumors cells, respectively. ${ }^{58} \mathrm{~A}$ further step in the investigation of the therapeutic impact of VISTA in breast cancer was made by Pilones et al, who tested the combination of local radiotherapy and various immunotherapy strategies using a murine mammary cancer model of metastatic and immune-resistant TNBC. ${ }^{59}$ They showed that the efficacy of an Ab specific for VISTA was enhanced by local radiotherapy, thus suggesting that a broad immunotherapeutic approach can help to increase durable responses in patients with TNBC. ${ }^{59}$

\section{Glioma}

Studies on immune checkpoint in gliomas are scarce. A preclinical study on animal models showed that VISTAdeficient animals were highly resistant to tumor induction in a murine brain glioma model, and depletion of CD4+ $\mathrm{T}$ cells, but not CD8+ $\mathrm{T}$ cells, promoted tumor formation. $^{28}$ These findings suggest that VISTA might have a potential immunomodulatory role also in the central nervous system.

\section{Renal Cell Carcinoma}

Despite the limited number of studies on VISTA in RCC, existing evidence supports an inhibitory role for VISTA in its immune environment. Clinical and pathological characteristics of patients with renal cancer cell (RCC) included in different studies have showed that VISTA is predominantly expressed in CD45+ cells in para-tumor and tumor tissues. VISTA is expressed in hematopoietic tissues and highly expressed within the myeloid compartment. $^{25,60} \mathrm{Ni}$ et al found that $\mathrm{T}$ cells coming from kidney cancer patients were activated following binding of a VISTA-Fc fusion protein to surface Fc receptors. ${ }^{61}$ In a different study Hong et al found out high prevalence of VISTA expression in clear cell RCC at both mRNA and protein levels. ${ }^{60}$ They also found out that CD14+ HLA-DR+ macrophages in tumors expressed higher levels of VISTA. The relationship between VISTA expression and CD8 $+\mathrm{T}$ cell responses identified in the study highlighted that VISTA may suppress tumor immunity.

\section{Ovarian and Endometrial Cancer}

Mulati et al showed that VISTA was expressed in 84 $(91.3 \%)$ of 92 ovarian cancer tissues samples, with no difference in survival. An important study was conducted by Liao et al who found that VISTA expression increased with advanced disease stage and lymph node metastasis, suggesting that VISTA expression might be involved in cancer progression.

Zong et al hypothesized that VISTA expression in ovarian tumor cells was associated with a favorable prognosis in patients with high-grade serious ovarian cancer and was related to pathological type and PD-L1

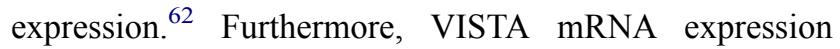
was positively related to immune escape-modulating genes. In vitro studies by Mulati et al showed that VISTA expression by tumor cells suppressed T cell proliferation and cytokine production resulting in immune evasion. ${ }^{63}$ These findings represent the first step to consider and explore VISTA as a potential candidate immunotherapeutic target in these cancers.

\section{Hematological Malignancies}

VISTA is predominantly expressed in the hematopoietic compartment, and particularly within the myeloid lineage. $^{25}$ Mice immunized with VISTA-Ig showed a suppressed proliferation of $\mathrm{T}$ cells and a weakened production of $\mathrm{T}$ cell cytokines and activation markers, suggesting the role of VISTA as a negative checkpoint regulator that suppresses $\mathrm{T}$ cell activation. ${ }^{25}$ Consistently, in a mouse model of acute myeloid leukemia (AML), the proliferation of tumor cells was reduced in VISTA-KO mice, supporting the hypothesis that VISTA induces immune evasion in AML. ${ }^{64}$ In addition, in the same study, VISTA Ab significantly decreased tumor cell growth in vivo in $\mathrm{KO}$ mice and extended their survival. ${ }^{64}$

One study conducted on human samples collected from patients affected by AML showed that VISTA was highly expressed on myeloid-derived suppressor cells in the peripheral blood. ${ }^{65}$ Moreover, VISTA expression was significantly higher in newly diagnosed AML patients compared to healthy controls. Importantly, knockdown of VISTA by specific siRNA potently reduced the inhibition of CD8 T cell activity. ${ }^{65}$ Taken together, these data suggest that 
VISTA may act as a suppressive modulator of antileukemia $\mathrm{T}$ cell response.

\section{Targeting Vista as a Treatment Strategy in Oncology: Clinical Data} JNJ-61,610,588 is a fully human $\operatorname{IgG1} \mathrm{mAb}$ directed against VISTA and has been the first one tested in humans. A phase I trial (ClinicalTrials.gov Identifier NCT02671955) was conducted among patients with different solid tumors, previously treated with at least one therapy for metastatic disease. The trial consisted of four parts: part 1 aimed to determine the maximum tolerated dose through a dose escalation mechanism, part 2 explored biomarkers among a cohort of patients with NSCLC, part 3 evaluated the recommended Phase 2 dose (RP2D) in participants with NSCLC, and part 4 evaluated the treatment at RP2D among a cohort of patients with different types of solid tumor (small-cell lung cancer, head and neck, pancreatic, colorectal, cervical cancer). Recruitment was completed in 2017 and results are still awaited.

Based on the comparative structural modeling and biological similarity between VISTA and other B7 family proteins, ${ }^{66}$ and on the different surface expression of VISTA from CTLA-4 and PD-(L) $1,{ }^{67}$ the strategy of simultaneously blocking multiple pathways was explored. Combination therapy (anti-VISTA and antiPD-1 or anti-PD-L1) enhanced T cell response and survival, and reduced tumor growth, thus supporting a synergic non-redundant mechanism of action to potentiate negative immune checkpoints blockade. ${ }^{67}$ CA-170 is an orally available small molecule that targets PD-L1/L2 and VISTA, resulting in T cell activation and cytokine production. CA-170 was previously tested in a phase I trial conducted in patients with advanced solid cancers and lymphoma already treated with standard therapies, including also anti-PD-(L)1 molecules (ClinicalTrial.gov NCT02812875). ${ }^{68}$ The doseescalation phase has shown acceptable safety results up to $2400 \mathrm{mg}$ daily dosage. Then, a dose expansion phase Ib evaluated the safety and tolerability of this drug only in patients with tumors expressing VISTA. In vitro, CA170 rescued $\mathrm{T}$ cell function similarly to PD- 1 antagonists, while inhibited the growth of mouse syngeneic tumors (B16 melanoma, CT26 and MC38 colon carcinoma). ${ }^{69}$ No dose limiting toxicities was observed in the phase Ia. A total of 59 patients were enrolled in the dose escalation phase (of whom, 22\% with NSCLC). Among the 50 evaluable patients, $50 \%(\mathrm{n}=25)$ showed stable disease and $16 \%(\mathrm{n}=8)$ tumor regression. About $20 \%$ of patients received at least 7 cycles of treatment, and 1 patient with follicular lymphoma experienced a stable disease at almost 2 years of treatment. In terms of safety, no dose limiting toxicities was observed. The majority of treatment-related adverse events (TRAEs) were grade $1 / 2$, consisting in fatigue, nausea, decreased appetite, vomiting, asthenia, constipation, cough, headache, pyrexia. A total of 5 patients experienced severe TRAEs of grade 3/4: lipase increased, pain, anemia, urinary infection and syncope. ${ }^{70}$

VISTA is highly expressed in pleural mesothelioma, leading to evaluate the activity of targeted agents against VISTA in this subgroup. ${ }^{71}$ In the cohort of metastatic pleural mesothelioma $(n=12)$ treated with CA-170 within the phase $\mathrm{Ib}$, no objective responses were reported, and 7 patients experienced a stable disease as best response. Among patients with stable disease, 2 received CA-170 $200 \mathrm{mg}$ twice daily with a median duration of response over 2 months, and 5 patients escalated to $1200 \mathrm{mg}$ twice daily with a median duration of response of 4 months. No new safety signals were observed, with low rates of drugrelated, immune-related adverse events (irAEs) or serious TRAEs. ${ }^{72}$

The Phase II open-label randomized trial (Clinical Trials Registry-India CTRI/2017/12/011026) compared CA-170 at the dosage of $400 \mathrm{mg}$ vs $800 \mathrm{mg}$ in 62 patients with multiple tumor types, already treated with one to three lines of therapies, excluding immune checkpoint inhibitors. The clinical benefit rate in the evaluable population $(\mathrm{n}=37)$ was $59.5 \%$, with a trend towards superior benefit at lower dosage. Overall, CA170 was well tolerated, but higher toxicity was reported in the $400 \mathrm{mg}$ group, with 8 patients experiencing irAEs: 5 with hypothyroidism, 2 with skin rash and one with grade 3 anemia and neutropenia, that led to treatment discontinuation. ${ }^{73}$

A sub-analysis conducted only among patients with NSCLC $(n=15)$ reported a disease control rate and a median PFS of $75 \%$ and 19.5 weeks in the $400 \mathrm{mg}$ group vs $50 \%$ and 7.9 weeks in the $800 \mathrm{mg}$ group, respectively. No objective responses, however, were observed in both arms. ${ }^{66}$

Table 2 summarizes the current clinical evidences on drugs targeting VISTA, and it describes about ongoing clinical trials. 
Table 2 Clinical Studies of Drugs Targeting VISTA

\begin{tabular}{|l|l|l|l|l|l|l|l|}
\hline $\begin{array}{l}\text { Clinical Trial } \\
\text { Number }\end{array}$ & Drug & Tumor Type & Phase & Setting & Design & $\begin{array}{l}\text { Actual } \\
\text { Status }\end{array}$ & $\begin{array}{l}\text { Summary } \\
\text { Results }\end{array}$ \\
\hline NCT0267I955 & $\begin{array}{l}\text { JNJ- } \\
61,610,588\end{array}$ & $\begin{array}{l}\text { NSCLC, SCLC, head } \\
\text { and neck, pancreatic, } \\
\text { colorectal, cervical } \\
\text { cancer }\end{array}$ & I & $\begin{array}{l}\text { Advanced } \\
\text { disease, >I line }\end{array}$ & Single-arm, open label & $\begin{array}{l}\text { Recruitment } \\
\text { stopped in } \\
2017\end{array}$ & $\begin{array}{l}\text { Results } \\
\text { awaited }\end{array}$ \\
\hline NCT028I2875 & CA-170 & $\begin{array}{l}\text { Solid tumors and } \\
\text { lymphoma }\end{array}$ & I & $\begin{array}{l}\text { Advanced } \\
\text { disease, >2 line }\end{array}$ & Single-arm, open label & $\begin{array}{l}\text { Enrolment } \\
\text { ongoing }\end{array}$ & $\begin{array}{l}\text { - Acceptable } \\
\text { safety up to } \\
2400 \text { mg daily } \\
\text { dosage } \\
-50 \% \text { stable } \\
\text { disease }\end{array}$ \\
\hline $\begin{array}{l}\text { CTRI/2017/12/ } \\
\text { 0II026 }\end{array}$ & CA-170 & $\begin{array}{l}\text { NSCLC, HN/oral } \\
\text { cavity, MSI-High or } \\
\text { dMMR cancers, HL }\end{array}$ & II & $\begin{array}{l}\text { Advanced } \\
\text { disease, } \\
\text { previously } \\
\text { treated with } \\
\text { I-3 lines }\end{array}$ & $\begin{array}{l}\text { Randomized, multiple- } \\
\text { arm, open label } \\
\text { (comparison between } \\
400 \text { mg vs } 800 \text { mg) }\end{array}$ & $\begin{array}{l}\text { Enrolment } \\
\text { ongoing } \\
\text { (estimated } \\
\text { I30 patients) }\end{array}$ & $\begin{array}{l}\text { lowards higher } \\
\text { activity with } \\
\text { lower dosage }\end{array}$ \\
\hline
\end{tabular}

Abbreviations: NSCLC, non-small-cell lung cancer; SCLC, small-cell-lung cancer; HN, head and neck; MSI, microsatellite instability; dMMR, mismatch repair deficient; HL, Hodgkin lymphoma; CBR, clinical benefit rate.

\section{Discussion and Conclusions}

In recent years, VISTA has been considered a potential biomarker in oncology, due to its role as a checkpoint regulator of the immune system. Several studies have investigated whether this immune checkpoint molecule had a prognostic implication in human solid tumors. A systematic review and meta-analysis of 10 studies $(2,440$ patients) correlated the value of VISTA with clinicopathological features and patient's outcome endpoints including OS and disease-specific survival (DSS), via pooled HR and 95\% confidence interval $(\mathrm{CI}) .{ }^{74}$ High expression of VISTA (cut-off differently defined among studies) was associated with better OS compared to low expression $(\mathrm{HR}=0.75,95 \%$ CI $0.66-0.86, \mathrm{p}<0.001)$, but no association with DSS was observed (HR $=1.57$, $95 \%$ CI $0.71-3.48, p=0.268$ ). Among clinicopathological characteristics, high expression of VISTA was significantly associated with high numbers of CD8+ TILs $($ risk ratio $=1.80,95 \%$ CI $1.41-2.31, \mathrm{p}<0.001) .{ }^{74} \mathrm{CD} 8+$ TILs have already been related to antitumoral properties in different studies involving patients with hepatocellular carcinoma, ${ }^{75}$ cervical and ovarian cancer, ${ }^{76-78}$ squamous-cell head and neck carcinoma, ${ }^{79}$ and lung cancer, ${ }^{80}$ thus potentially indirectly supporting the positive prognostic role of VISTA as a biomarker.
In conclusion, we provided an updated overview on the value of VISTA as a biomarker for cancer immunotherapy, reporting its expression in different cancer types, its prognostic role and the rationale for its targeting in clinical practice. Currently, small molecules or mAb directed against VISTA have demonstrated to have acceptable tolerability profiles and clinical activity. Nevertheless, the complexity of VISTA pathway, along with some unclear biological aspects, limit its applicability as a target for cancer immunotherapy in the near future. A deeper characterization of this immune checkpoint may help in defining its role within immune signatures of solid and hematological malignancies, and to design future therapeutic strategies.

\section{Funding}

This work received no funding.

\section{Disclosure}

Dr Tagliamento reported travel grants from Roche, BristolMyers Squibb, AstraZeneca, Takeda and honoraria as medical writer from Novartis and Amgen outside the submitted work. Dr Brandão reports a travel grant and speaker honorarium from Roche outside the submitted work. Dr. Alfredo Addeo reports personal fees from BMS and 
advisory for BMS, AstraZeneca, Roche, Astella, Pfizer, MSD, and Eli Lilly, outside the submitted work. Dr Lambertini acted as a consultant/advisor for Roche, Lilly, Novartis, and AstraZeneca, and received honoraria from Sandoz, Roche, Lilly, Pfizer, and Novartis, and speaker Honoraria from Takeda, outside the submitted work. The other authors reported no other potential conflicts of interest for this work.

\section{References}

1. $\mathrm{He} \mathrm{X}, \mathrm{Xu}$ C. Immune checkpoint signaling and cancer immunotherapy. Cell Res. 2020;30(8):660-669. doi:10.1038/s41422020-0343-4

2. Darvin P, Toor SM, Sasidharan Nair V, Elkord E. Immune checkpoint inhibitors: recent progress and potential biomarkers. Exp Mol Med. 2018;50(12):1-11. doi:10.1038/s12276-018-0191-1

3. Xu W, Hiếu T, Malarkannan S, Wang L. The structure, expression, and multifaceted role of immune-checkpoint protein VISTA as a critical regulator of anti-tumor immunity, autoimmunity, and inflammation. Cell Mol Immunol. 2018;15(5):438-446. doi:10.1038/ cmi.2017.148

4. De Sousa Linhares A, Leitner J, Grabmeier-Pfistershammer K, Steinberger P. Not all immune checkpoints are created equal. Front Immunol. 2018;9:1909. doi:10.3389/fimmu.2018.01909

5. Torphy R, Schulick R, Zhu Y. Newly emerging immune checkpoints: promises for future cancer therapy. Int J Mol Sci. 2017;18(12):2642. doi:10.3390/ijms 18122642

6. Lambertini M, Preusser M, Zielinski CC. New emerging targets in cancer immunotherapy beyond CTLA-4, PD-1 and PD-L1: introducing an "ESMO open - cancer horizons" series. ESMO Open. 2019;4 (Supp13):e000501. doi:10.1136/esmoopen-2019-000501

7. Rossi G, Russo A, Tagliamento M, et al. Precision medicine for NSCLC in the era of immunotherapy: new biomarkers to select the most suitable treatment or the most suitable patient. Cancers. 2020;12(5):1125. doi:10.3390/cancers12051125

8. Rebuzzi SE, Zullo L, Rossi G, et al. Novel emerging molecular targets in non-small cell lung cancer. Int J Mol Sci. 2021;22 (5):2625. doi:10.3390/ijms22052625

9. Ruffo E, Wu RC, Bruno TC, Workman CJ, Vignali DAA. Lymphocyte-activation gene 3 (LAG3): the next immune checkpoint receptor. Semin Immunol. 2019;42:101305. doi:10.1016/j. smim.2019.101305

10. Solinas C, Aiello M, Rozali E, Lambertini M, Willard-Gallo K, Migliori E. Programmed cell death-ligand 2: a neglected but important target in the immune response to cancer? Transl Oncol. 2020;13 (10):100811. doi:10.1016/j.tranon.2020.100811

11. Pardoll DM. The blockade of immune checkpoints in cancer immunotherapy. Nat Rev Cancer. 2012;12(4):252-264. doi:10.1038/ $\operatorname{nrc} 3239$

12. Friedlaender A, Addeo A, Banna G. New emerging targets in cancer immunotherapy: the role of TIM3. ESMO Open. 2019;4:e000497. doi:10.1136/esmoopen-2019-000497

13. Acharya N, Sabatos-Peyton C, Anderson AC. Tim-3 finds its place in the cancer immunotherapy landscape. J Immunother Cancer. 2020;8 (1):e000911. doi:10.1136/jitc-2020-000911

14. Chauvin J-M, Zarour HM. TIGIT in cancer immunotherapy. $J$ Immunother Cancer. 2020;8(2):e000957. doi:10.1136/jitc-2020000957

15. Yang S, Wei W, Zhao Q. B7-H3, a checkpoint molecule, as a target for cancer immunotherapy. Int J Biol Sci. 2020;16(11):1767-1773. doi:10.7150/ijbs.41105
16. Genova C, Boccardo S, Mora M, et al. Correlation between B7-H4 and survival of non-small-cell lung cancer patients treated with nivolumab. J Clin Med. 2019;8(10):1566. doi:10.3390/jcm8101566

17. Tagliamento M, Bironzo P, Novello S. New emerging targets in cancer immunotherapy: the role of VISTA. ESMO Open. 2019;4: e000683. doi:10.1136/esmoopen-2020-000683

18. De Mattos-arruda L, Blanco-Heredia J, Aguilar-Gurrieri C, Carrillo J, Blanco J. New emerging targets in cancer immunotherapy: the role of neoantigens. ESMO Open. 2019;4:e000684. doi:10.1136/esmoopen2020-000684

19. ElTanbouly MA, Schaafsma E, Noelle RJ, Lines JL. VISTA: coming of age as a multi-lineage immune checkpoint. Clin Exp Immunol. 2020;200(2):120-130. doi:10.1111/cei.13415

20. Huang X, Zhang X, Li E, et al. VISTA: an immune regulatory protein checking tumor and immune cells in cancer immunotherapy. J Hematol Oncol. 2020;13(1):83. doi:10.1186/s13045-020-00917-y

21. Wang L, Rubinstein R, Lines JL, et al. VISTA, a novel mouse Ig superfamily ligand that negatively regulates $\mathrm{T}$ cell responses. $J$ Exp Med. 2011;208(3):577-592. doi:10.1084/jem.20100619

22. Aloia L, Parisi S, Fusco L, Pastore L, Russo T. Differentiation of embryonic stem cells 1 (Dies1) is a component of Bone Morphogenetic Protein 4 (BMP4) signaling pathway required for proper differentiation of mouse embryonic stem cells. J Biol Chem. 2010;285(10):7776-7783. doi:10.1074/jbc.M109.077156

23. Battista M, Musto A, Navarra A, Minopoli G, Russo T, Parisi S. miR$125 \mathrm{~b}$ regulates the early steps of ESC differentiation through dies 1 in a TGF-independent manner. Int J Mol Sci. 2013;14(7):13482-13496. doi:10.3390/ijms140713482

24. Flies DB, Wang S, Xu H, Chen L. Cutting edge: a monoclonal antibody specific for the programmed death-1 homolog prevents graft-versus-host disease in mouse models. J Immunol. 2011;187 (4):1537-1541. doi:10.4049/jimmunol.1100660

25. Lines JL, Pantazi E, Mak J, et al. VISTA is an immune checkpoint molecule for human T cells. Cancer Res. 2014;74(7):1924-1932. doi:10.1158/0008-5472.CAN-13-1504

26. Bharaj P, Chahar HS, Alozie OK, et al. Characterization of Programmed Death-1 Homologue-1 (PD-1H) expression and function in normal and HIV infected individuals. Kaveri SV, ed. PLoS One. 2014;9(10):e109103. doi:10.1371/journal.pone.0109103

27. Wang L, Le Mercier I, Putra J, et al. Disruption of the immune-checkpoint VISTA gene imparts a proinflammatory phenotype with predisposition to the development of autoimmunity. Proc Natl Acad Sci. 2014;111(41):14846-14851. doi:10.1073/ pnas. 1407447111

28. Flies DB, Han X, Higuchi T, et al. Coinhibitory receptor PD-1H preferentially suppresses $\mathrm{CD}^{+} \mathrm{T}$ cell-mediated immunity. $J$ Clin Invest. 2014;124(5):1966-1975. doi:10.1172/JCI74589

29. Flies DB, Higuchi T, Chen L. Mechanistic assessment of PD-1H coinhibitory receptor-induced $\mathrm{T}$ cell tolerance to allogeneic antigens. J Immunol. 2015;194(11):5294-5304. doi:10.4049/jimmunol.1402648

30. Liu J, Yuan Y, Chen W, et al. Immune-checkpoint proteins VISTA and PD-1 nonredundantly regulate murine T-cell responses. Proc Natl Acad Sci. 2015;112(21):6682LP- 6687. doi:10.1073/pnas.1420370112

31. Tham EL, Freeley SJ, Bearder S, et al. VISTA deficiency protects from immune complex-mediated glomerulonephritis by inhibiting neutrophil activation. J Autoimmun. 2020;113:102501. doi:10.1016/ j.jaut.2020.102501

32. Hid Cadena R, Reitsema RD, Huitema MG, et al. Decreased expression of negative immune checkpoint VISTA by CD4+ T cells facilitates $\mathrm{T}$ helper $1, \mathrm{~T}$ helper 17 , and $\mathrm{T}$ follicular helper lineage differentiation in GCA. Front Immunol. 2019;10:1638. doi:10.3389/ fimmu.2019.01638

33. Borggrewe M, Grit C, Den dunnen WFA, et al. VISTA expression by microglia decreases during inflammation and is differentially regulated in CNS diseases. Glia. 2018;66(12):2645-2658. doi:10.1002/ glia.23517 
34. ElTanbouly MA, Schaafsma E, Smits NC, et al. VISTA re-programs macrophage biology through the combined regulation of tolerance and anti-inflammatory pathways. Front Immunol. 2020;11:580187. doi:10.3389/fimmu.2020.580187

35. Wang J, Wu G, Manick B, et al. VSIG-3 as a ligand of VISTA inhibits human T-cell function. Immunology. 2019;156(1):74-85. doi:10.1111/imm.13001

36. Buchbinder EI. Immune checkpoint therapies for melanoma. Hematol Oncol Clin North Am. 2021;35(1):99-109. doi:10.1016/j. hoc. 2020.08 .013

37. Rosenbaum SR, Knecht M, Mollaee M, et al. FOXD3 regulates VISTA expression in melanoma. Cell Rep. 2020;30(2):510-524.e6. doi:10.1016/j.celrep.2019.12.036

38. Xu W, Dong J, Zheng Y, et al. Immune-checkpoint protein VISTA regulates antitumor immunity by controlling myeloid cell-mediated inflammation and immunosuppression. Cancer Immunol Res. 2019;7 (9):1497LP- 1510. doi:10.1158/2326-6066.CIR-18-0489

39. Kakavand H, Jackett LA, Menzies AM, et al. Negative immune checkpoint regulation by VISTA: a mechanism of acquired resistance to anti-PD-1 therapy in metastatic melanoma patients. Mod Pathol off J US Can Acad Pathol Inc. 2017;30(12):1666-1676. doi:10.1038/ modpathol.2017.89

40. Kuklinski LF, Yan S, Li Z, et al. VISTA expression on tumor-infiltrating inflammatory cells in primary cutaneous melanoma correlates with poor disease-specific survival. Cancer Immunol Immunother. 2018;67(7):1113-1121. doi:10.1007/s00262-018-2169-1

41. Villarroel-Espindola F, Yu X, Datar I, et al. Spatially resolved and quantitative analysis of VISTA/PD-1H as a novel immunotherapy target in human non-small cell lung cancer. Clin Cancer Res. 2018;24(7):1562-1573. doi:10.1158/1078-0432.CCR-17-2542

42. Brcic L, Stanzer S, Krenbek D, et al. Immune cell landscape in therapynaïve squamous cell and adenocarcinomas of the lung. Virchows Arch 2018;472(4):589-598. doi:10.1007/s00428-018-2326-0

43. Ladanyi M, Robinson BW, Campbell PJ; for the TCGA Research Network. The TCGA malignant pleural mesothelioma (MPM) project: VISTA expression and delineation of a novel clinical-molecular subtype of MPM. J Clin Oncol. 2018;36(15_suppl):8516. doi:10.1200/JCO.2018.36.15_suppl.8516

44. Muller S, Victoria Lai $\bar{W}$, Adusumilli PS, et al. V-domain Ig-containing suppressor of T-cell activation (VISTA), a potentially targetable immune checkpoint molecule, is highly expressed in epithelioid malignant pleural mesothelioma. Mod Pathol. 2020;33 (2):303-311. doi:10.1038/s41379-019-0364-z

45. Rooney C, Nixon C, Blyth K, Sethi T, Murphy D, McCaughan F. S45 VISTA expression in malignant pleural mesothelioma. Novel Insights into Malignant Pleural Disease. Thorax. 2019:74:A29.2-A30. doi:10.1136/thorax-2019-BTSabstracts2019.51

46. Liu J, Xie X, Xuan C, et al. High-density infiltration of V-domain immunoglobulin suppressor of T-cell activation up-regulated immune cells in human pancreatic cancer. Pancreas. 2018;47(6):725-731. doi:10.1097/mpa.0000000000001059

47. Byers JT, Paniccia A, Kaplan J, et al. Expression of the novel costimulatory molecule B7-H5 in pancreatic cancer. Ann Surg Oncol. 2015;22(3):1574-1579. doi:10.1245/s10434-014-4293-2

48. Blando J, Sharma A, Higa MG, et al. Comparison of immune infiltrates in melanoma and pancreatic cancer highlights VISTA as a potential target in pancreatic cancer. Proc Natl Acad Sci U S A. 2019;116(5):1692-1697. doi:10.1073/pnas.1811067116

49. Xie S, Huang J, Qiao Q, et al. Expression of the inhibitory B7 family molecule VISTA in human colorectal carcinoma tumors. Cancer Immunol Immunother. 2018;67(11):1685-1694. doi:10.1007/s00262-018-2227-8

50. Zaravinos A, Roufas C, Nagara M, et al. Cytolytic activity correlates with the mutational burden and deregulated expression of immune checkpoints in colorectal cancer. J Exp Clin Cancer Res. 2019;38 (1):364. doi:10.1186/s13046-019-1372-z
51. Alexandrov LB, Nik-Zainal S, Wedge DC, et al. Signatures of mutational processes in human cancer. Nature. 2013;500(7463):415-421. doi:10.1038/nature 12477

52. Xie X, Zhang J, Shi Z, et al. The expression pattern and clinical significance of the immune checkpoint regulator VISTA in human breast cancer. Front Immunol. 2020;11:2808. doi:10.3389/fimmu.2020.563044

53. Zong L, Mo S, Yu S, et al. Expression of the immune checkpoint VISTA in breast cancer. Cancer Immunol Immunother. 2020;69 (8):1437-1446. doi:10.1007/s00262-020-02554-3

54. Stanton SE, Adams S, Disis ML. Variation in the incidence and magnitude of tumor-infiltrating lymphocytes in breast cancer subtypes: a systematic review. JAMA Oncol. 2016;2(10):1354-1360. doi:10.1001/jamaoncol.2016.1061

55. Solinas C, Carbognin L, De Silva P, Criscitiello C, Lambertini M. Tumor-infiltrating lymphocytes in breast cancer according to tumor subtype: current state of the art. Breast. 2017;35:142-150. doi:10.1016/j.breast.2017.07.005

56. Losurdo A, De Sanctis R, Fernandes B, et al. Insights for the application of TILs and AR in the treatment of TNBC in routine clinical practice. Sci Rep. 2020;10(1):20100. doi:10.1038/s41598-020-77043-9

57. Agostinetto E, Eiger D, Punie K, de Azambuja E. Emerging therapeutics for patients with triple-negative breast cancer. Curr Oncol Rep. 2021;23(5):57. doi:10.1007/s11912-021-01038-6

58. Cao X, Ren X, Zhou Y, et al. VISTA expression on immune cells correlates with favorable prognosis in patients with triple-negative breast cancer. Front Oncol. 2021;10:3010. doi:10.3389/fonc.2020.583966

59. Pilones KA, Hensler M, Daviaud C, et al. Converging focal radiation and immunotherapy in a preclinical model of triple negative breast cancer: contribution of VISTA blockade. OncoImmunology. 2020;9 (1):1830524. doi:10.1080/2162402X.2020.1830524

60. Hong S, Yuan Q, Xia H, et al. Analysis of VISTA expression and function in renal cell carcinoma highlights VISTA as a potential target for immunotherapy. Protein Cell. 2019;10(11):840-845. doi:10.1007/s13238-019-0642-z

61. Ni L, Dong C. New checkpoints in cancer immunotherapy. Immunol Rev. 2017;276(1):52-65. doi:10.1111/imr.12524

62. Zong L, Zhou Y, Zhang M, Chen J, Xiang Y. VISTA expression is associated with a favorable prognosis in patients with high-grade serous ovarian cancer. Cancer Immunol Immunother. 2020;69 (1):33-42. doi:10.1007/s00262-019-02434-5

63. Mulati K, Hamanishi J, Matsumura N, et al. VISTA expressed in tumour cells regulates $\mathrm{T}$ cell function. Br $J$ Cancer. 2019;120 (1):115-127. doi:10.1038/s41416-018-0313-5

64. Kim TK, Han X, Wang J, et al. PD-1H (VISTA) induces immune evasion in acute myeloid leukemia. Blood. 2017;130 (Supplement1):2658. doi:10.1182/blood.V130.Suppl_1.2658.2658

65. Wang L, Jia B, Claxton DF, et al. VISTA is highly expressed on MDSCs and mediates an inhibition of T cell response in patients with AML. Oncoimmunology. 2018;7(9):e1469594. doi:10.1080/ 2162402X.2018.1469594

66. Musielak B, Kocik J, Skalniak L, et al. CA-170 - a potent smallmolecule PD-L1 inhibitor or not? Molecules. 2019;24(15):2804. doi:10.3390/molecules24152804

67. Deng J, Le Mercier I, Kuta A, Noelle RJ, New A. VISTA on combination therapy for negative checkpoint regulator blockade. J Immunother Cancer. 2016;4(1):86. doi:10.1186/s40425-016-0190-5

68. Lee JJ, Powderly JD, Patel MR, et al. Phase 1 trial of CA-170, a novel oral small molecule dual inhibitor of immune checkpoints PD-1 and VISTA, in patients (pts) with advanced solid tumor or lymphomas. J Clin Oncol. 2017;35(15_suppl):TPS3099-TPS3099. doi:10.1200/JCO.2017.35.15_suppl.TPS3099

69. Powderly J, Patel MR, Lee JJ, et al. CA-170, a first in class oral small molecule dual inhibitor of immune checkpoints PD-L1 and VISTA, demonstrates tumor growth inhibition in pre-clinical models and promotes T cell activation in phase 1 study. Ann Oncol. 2017;28: v405-v406. doi:10.1093/annonc/mdx376.007 
70. Bang Y-L, Sosman JA, Daud A, et al. Phase 1 study of CA-170, a first-in-class, orally available, small molecule immune checkpoint inhibitor (ICI) dually targeting VISTA and PD-L1, in patients with advanced solid tumors or lymphomas. in P341. (2018) 6:175. $J$ Immunother Cancer. 2018;6(S1):114, s40425-018-0422-y. doi:10.1186/s40425-018-0422-y

71. Zauderer M, Brody J, Marron T, et al. P2.06-07 phase 1 study of CA-170: first-in-class small molecule targeting VISTA/PD-L1 in patients with malignant pleural mesothelioma. J Thorac Oncol. 2019;14(10):S757-S758. doi:10.1016/j.jtho.2019.08.1625

72. Zauderer M, Brody J, Marron T, et al. First-in-class small molecule CA-170 targeting VISTA: a report on efficacy outcomes from a cohort of 12 malignant pleural mesothelioma (MPM) patients in study CA- 170-101. J Immunother Cancer 2019;7:O28. $J$ Immunother Cancer. 2019;7(S1):283, s40425-019-0764-0. doi:10.1186/s40425-019-0764-0

73. Radhakrishnan VS, Bakhshi S, Prabhash K, et al. Phase 2 trial of CA-170, a novel oral small molecule dual inhibitor of immune checkpoints VISTA and PD-1, in patients (pts) with advanced solid tumor and Hodgkin lymphoma. J Immunother Cancer 2018;6:P714. J Immunother Cancer. 2018;6(S2):1-13. doi:10.1186/s40425-018-0434-7

74. He X-L, Zhou Y, Lu H-Z, Li Q-X, Wang Z. Prognostic value of VISTA in solid tumours: a systematic review and meta-analysis. Sci Rep. 2020;10(1):2662. doi:10.1038/s41598-020-59608-w

75. Gabrielson A, Wu Y, Wang H, et al. Intratumoral CD3 and CD8 T-cell densities associated with relapse-free survival in HCC. Cancer Immunol Res. 2016;4(5):419-430. doi:10.1158/2326-6066.CIR-15-0110
76. Liang Y, Lü W, Zhang X, Lü B. Tumor-infiltrating CD8+ and FOXP3+ lymphocytes before and after neoadjuvant chemotherapy in cervical cancer. Diagn Pathol. 2018;13(1):93. doi:10.1186/ s13000-018-0770-4

77. Li J, Wang J, Chen R, Bai Y, Lu X. The prognostic value of tumor-infiltrating $\mathrm{T}$ lymphocytes in ovarian cancer. Oncotarget. 2017;8(9):15621-15631. doi:10.18632/oncotarget.14919

78. Goode EL, Block MS, Kalli KR, et al; Ovarian Tumor Tissue Analysis (OTTA) Consortium. Dose-response association of CD8 +tumor-infiltrating lymphocytes and survival time in high-grade serous ovarian cancer. JAMA Oncol. 2017;3(12):e173290. doi:10.1001/ jamaoncol.2017.3290

79. Nguyen N, Bellile E, Thomas D, et al. Tumor infiltrating lymphocytes and survival in patients with head and neck squamous cell carcinoma. Head Neck. 2016;38(7):1074-1084. doi:10.1002/ hed. 24406

80. Ye S-L, Li X-Y, Zhao K, Feng T. High expression of CD8 predicts favorable prognosis in patients with lung adenocarcinoma: a cohort study. Medicine. 2017;96(15):e6472. doi:10.1097/MD.00000 00000006472

81. Chung YS, Kim M, Cha YJ, Kim KA, Shim HS. Expression of V-set immunoregulatory receptor in malignant mesothelioma. Mod Pathol. 2020;33(2):263-270. doi:10.1038/s41379-019-0328-3
ImmunoTargets and Therapy

\section{Publish your work in this journal}

ImmunoTargets and Therapy is an international, peer-reviewed open access journal focusing on the immunological basis of diseases, potential targets for immune based therapy and treatment protocols employed to improve patient management. Basic immunology and physiology of the immune system in health, and disease will be also covered. In addition, the journal will focus on the impact of management

\section{Dovepress}

programs and new therapeutic agents and protocols on patient perspectives such as quality of life, adherence and satisfaction. The manuscript management system is completely online and includes a very quick and fair peer-review system, which is all easy to use. Visit http://www.dovepress.com/testimonials.php to read real quotes from published authors. 